\title{
The Speed of Light in a Novel Gravitational Environment
}

\author{
Gianni Donati \\ Associazione Italiana di Ingegneria Chimica, Milano, Italy \\ Email: gia.donati@tiscali.it
}

How to cite this paper: Donati, G. (2017) The Speed of Light in a Novel Gravitational Environment. Journal of Applied Mathematics and Physics, 5, 1938-1950. https://doi.org/10.4236/jamp.2017.510164

Received: September 20, 2017

Accepted: October 14, 2017

Published: October 17, 2017

Copyright $\odot 2017$ by author and Scientific Research Publishing Inc. This work is licensed under the Creative Commons Attribution International License (CC BY 4.0).

http://creativecommons.org/licenses/by/4.0/

\begin{abstract}
In this paper, a review of the experiments, developments and ideas which have been presented on this topic together with its natural extension to the description of gravity, the ultimate dominating mystery of the universe, was taken out. A parallel mechanism was proposed between the emission of light and of neutrino by quantum leaps of electrons between the fixed energy levels of atomic orbits. The analysis of the neutron-proton mix of existing nuclides provides a rule for the calculation of the neutrino flux from matter and suggests both a medium for the transmission of light and a solution to the problem of gravitation.
\end{abstract}

\section{Keywords}

Light, Gravity, Nuclear Structure, Neutrinos, Relativity

\section{Introduction}

The knowledge from astronomical data that light can pass through space practically devoid of matter led physicists in the 19th century to postulate the existence of a medium in which light waves could be propagated.

This medium, called the ether, was supposed to be a weightless stationary substance permeating the entire universe.

If the Earth travels in the stationary ether with a speed $v$ and light waves with a speed $c$, it should be possible to devise an experiment from which the absolute speed of Earth could be deduced.

It is important to revise the follow up of this idea because it led, through many contradictions and dilemmas, to the greatest revolution in physics of the 20th century.

However, even today, these contradictions and dilemmas are far to be solved 
and a lot of objections on the logic of the scientific path followed are appearing in the literature.

Coming back to our experiment, it was supposed that, if light is emitted in the direction of the motion of the Earth, its velocity relative to the Earth will be $-v$, while, travelling in the opposite direction, we will have a velocity equal to $c+v$.

However, early in 1825, Fresnel introduced for motion in a medium and in particular in a stationary ether the concept of ether drag $\alpha=\left(1-n^{-2}\right)$ were $n$ is the refractive index.

$$
c^{*} \approx c / n \mp v\left(1-1 / n^{2}\right)
$$

Formula (1) received experimental confirmation by Fizeau in 1852 and cannot be reconciled with the mechanical addition of velocity supposed for the experiment.

In fact, if $n=1$ and $\alpha=0 \quad c^{*}=c$, that is the velocity $c=$ constant, according to the hypothesis that in the ether without particles (vacuum) there should be the isotropy of the speed of light.

On the other extreme, if $n=\infty$ and $\alpha=1$, we have $c^{*}=\mp v$.

Michelson and Morley [1] made in 1887 their famous experiment with an ingenious interferometer ignoring Fresnel and using the mechanical addition of velocities.

The interferometer has two arms, one in the direction of $v$ and the other perpendicular to it; a beam of light is divided in two perpendicular beams by a half-silvered mirror, reflected back along the arms and then collected by a photographic plate where an interference is expected due to the slightly different times $t_{1}$ and $t_{2}$ of arrival of the two beams.

If the path length is $L$ and equal in the two directions we have:

$$
t_{1}-t_{2}=\frac{2 L}{c}\left(\frac{1}{1-\left(\frac{v^{2}}{c^{2}}\right)}-\frac{1}{\sqrt{1-\left(\frac{v^{2}}{c^{2}}\right)}}\right) \cong \frac{L}{c}\left(\frac{v^{2}}{c^{2}}\right)
$$

and if the interferometer is rotated $90^{\circ}$ the time difference doubles and, with a light having wave length $\lambda$, the ratio of displacement $2 c\left(t_{1}-t_{2}\right)$ to wavelength is given by:

$$
A m=\frac{2 L}{\lambda}\left(\frac{v^{2}}{c^{2}}\right)
$$

In the experiment no significant displacement was observed.

The simplest explanation was that the Earth carried the ether with it, and that at the time seemed unattractive for two reasons: first the ether was not stationary and second the velocity of light would depend on the velocity of its source carrying the ether.

Fitzgerald (1893) introduced the hypothesis that when a body travels in a di- 
rection parallel to the Earth motion contracts in length by a factor $\sqrt{1-\left(\frac{v^{2}}{c^{2}}\right)}$ but no contraction occurs in the perpendicular direction.

With this artifact, light did not need to travel faster in the parallel direction than in the perpendicular one and the stationary ether was preserved.

Lorentz found it useful to adopt Fitzgerald contraction to the radius of the electron in motion with velocity $v$ and found the increase of electron mass from the rest mass $m_{o}$ to $m$ :

$$
m=m_{o} / \sqrt{1-\left(\frac{v^{2}}{c^{2}}\right)}
$$

Lorentz went further when he found that classical transformations are not invariant for electromagnetic phenomena and derived the following transformations for a system $S^{\prime}$ in motion with constant velocity $V$ along $x$ axis of a reference system $S$ :

$$
\begin{aligned}
& x^{\prime}=(x-v t) / \sqrt{1-\left(\frac{v^{2}}{c^{2}}\right)} \\
& y^{\prime}=y \\
& z^{\prime}=z \\
& t^{\prime}=\left(t-\frac{v x}{c^{2}}\right) / \sqrt{1-\left(\frac{v^{2}}{c^{2}}\right)}
\end{aligned}
$$

Light is an electromagnetic wave and recently Demjanov [2] [3] warned about the use of the classical addition of velocity for interpreting Michelson and Morley's experiment and all the experimental investigations and refinements that followed.

He shows how the Fresnel Equation (1) represents the first order approximation of the Lorentz invariant transformations and questions about the existence of a real vacuum.

He measures the amplitude of the fringe shift for various media filling the interferometer in function of the polarizability of the particles $\Delta \epsilon=n^{2}-1$ and polarizability of stationary ether $\Delta \varepsilon=1$ with the refractive index of the total media plus ether $n=\sqrt{1+\Delta \varepsilon}$.

He defines the amplitude of the fringe shift in function of $\Delta \varepsilon$ :

$$
A m=\frac{2 L}{\lambda \sqrt{\varepsilon}} \frac{v^{2}}{c^{2}} \Delta \varepsilon(1-\Delta \epsilon)
$$

Equation (6) is similar to (2), but takes into account the nature and pressure of the gas filling the interferometer.

He revises all the components of Michelson and Morley interferometer (glass plates, reflecting mirrors etc.) as source of errors and comes to the conclusion that the ether wind has been underestimated by them and by all subsequent authors.

This topic is under discussion by several even more recent authors. 
On the contrary, Shaff [4] in 2012 agreed on the null or at least modest results of Michelson and Morley's experiments but thought that this is due to the ether not being stationary but moving around solid bodies (the Sun, the Earth) in a Keplerian motion.

He stated that the anisotropy of light can be demonstrated with the help of global positioning system (GPS); the postulates of special relativity are therefore invalidated by experimental measurements of the one way velocity of light and a new theory of gravitation can be developed in which a physical media, the rotating ether, and not a geometrical void space, moves the planets around the Sun.

Apart from this revival of ether and its curious role in gravitation, other authors make use of GPS to demonstrate light anisotropy.

In 2012, Gift [5] discussed about clocks synchronization techniques and the differences in times and hence in light speed between westward and eastward transmission due to Earth rotation.

A modified Michelson-Morley experiment using GPS clocks is presented and the changed East-West light speed arising from the ether drift is claimed.

Schreiber [6] added additional criticism on Michelson-Morley analysis for neglecting Fizeau Equation (1) and on the definition of vacuum, an unreal thing with nothing in it.

Croca [7] added to the discussion the old claim of Poincarè (1898) of the physical impossibility of measuring the one way velocity of light using two separate clocks and proposes an interferometer with only one clock and two go-return legs, one go leg filled with an optical medium of known refractive index.

From these examples, we can conclude that from the 19th century till now nobody knows what this ether is; whether it is something that allows the transmission of light or a sort of dark matter to explain the unknown phenomena of gravitation.

When Einstein took the problem in his hands (1905), he had to solve the question mark raised by Michelson and Morley experiment and maintain the invariance of laws with respect to coordinates transformations.

To develop the theory of special relativity, he started with two postulates:

The first states that the laws of physics are the same in two systems moving at constant relative velocity.

The second states that the velocity of light is the same in free space for all observers independently of the velocity of the source and of the observer,

From these postulates, the Lorentz transformation Equation (5) can be deduced as other kinematic relationships.

It was not until 1916, with the general theory of relativity that he made a jump from light to gravity and he was able to show that physical laws can be expressed in a form that is valid for any choice of space-time coordinates.

He eliminated ether, substituting it with vacuum completely devoid of matter, but not all physicians were happy because they thought it was an artifact and, as we have seen, even today some of them are inventing other artifacts to maintain the ether. 
In 1911, Einstein published the first paper [8] on the influence of gravitation on the propagation of light and in 1916 he presented [9] a complete version of his general theory of relativity in which 1911 errors where corrected.

In synthesis, given two points at distances $R_{1}$ and $R_{2}$ from a center of gravity $\left(R_{2}>R_{1}\right)$ of a mass $M$, the energy of a beam of light increases from $R_{2}$ to $R_{1}$ following the gravitational potential $\varnothing$ and so does the frequency:

$$
f_{1}=f_{2} \sqrt{\frac{1-2 \frac{\varnothing_{2}}{c^{2}}}{1-2 \frac{\varnothing_{1}}{c^{2}}}}=f_{2} \sqrt{\frac{1-\frac{2 G M}{R_{2} c^{2}}}{1-\frac{2 G M}{R_{1} c^{2}}}}
$$

with $G$ the universal gravitational constant.

Therefore light from $R_{2}$ to $R_{1}$ is blue-shifted and, in a first approximation, Einstein computed that light coming from the Sun is red-shifted

$$
f=f_{o}\left(1+2 \frac{\varnothing}{c^{2}}\right) \text { or } \frac{f_{o}-f}{f_{o}}=-\frac{\varnothing}{c^{2}} \cong 4 \times 10^{-6}
$$

Einstein then shows that the clocks in $R_{1}$ must run slower than in $R_{2}$ and distances shrink the same proportion in a way that the velocity of light measured in $R_{1}$ and $R_{2}$ is the same and equal to $c$.

If however we measure the velocity of light of $R_{2}$ with the metric of $R_{1}$ we find that:

$$
c_{2}=c_{1} \sqrt{\frac{1+\frac{2 G M}{R_{1} c^{2}}}{1+\frac{2 G M}{R_{2} c^{2}}}}
$$

This is how the constancy of velocity of light is maintained, in a different way than in special relativity, and Einstein could arrive to the conclusion that the velocity of light in a gravitational field is a function of the place and we can infer that light rays propagated across a gravitational field undergo deflection.

We can compute the angle of deflection of light as $\alpha=\frac{4 G M}{c^{2} R}$, in agreement with astronomical measurements.

To test Einstein's theory of General Relativity in 1959 Pound and Rebka [10] proposed an experiment in which a photon, emitted from a source travelling in a gravitational field is red shifted; but, if the emitting source moves the right speed relative to the receiver, the Doppler shift will cancel the gravitational field.

$$
\sqrt{\frac{1-v / c}{1+v / c} \frac{1+\frac{2 G M}{R_{1} c^{2}}}{1+\frac{2 G M}{R_{2} c^{2}}}}=1
$$

This, together with astronomical measurements of light deflection, confirmed the effect of gravity on propagation of light.

We can see that light and gravity are strictly related and all mentioned criti- 
cisms on the isotropy of light and the rumors that fill some unconventional papers even today are aimed to attach and possibly invalidate the special and general theory of relativity.

However, we have to recognize that during the last century till now no other theory has been formulated having the rigor and the maturity of Einstein relativity.

He noticed that Newton's expression for the gravitational force between two objects depends on the masses and on the distance separating the bodies, but makes no mention of time at all. In this view of the world, if one mass is moved, the other perceives the change (as a decrease or increase of the gravitational force) instantaneously. If exactly true this would be a physical effect which travels faster than light (in fact, at infinite speed), and would be inconsistent with the Special Theory of Relativity.

This may not be completely true if we change our view of gravity, but, as a consequence, he developed an equation to implement Newton Law in line with the formulation of Maxwell for electrodynamics.

We have to confess that we are not confident with the use of Einstein field equations for the solution of gravitational problems: despite their simple appearance they are actually quite complicated and, similarly to Maxwell equations, they have been solved only for very simple cases.

For example, there is no known complete solution for a space time with two massive bodies, while they have been used by Einstein followers and, against his opinion, for the prediction of black holes and for hypothesizing the birth and the evolution of the universe.

Einstein strongly believed that black holes did not exist and that the cosmological constant introduced in his field equations for predicting the expansion of the universe was an error and he continued to resist to the idea of a Big Bang, which he at first found "abominable", even though other theoreticians had shown it to be a natural consequence of his general theory of relativity.

Unfortunately, the idea of the Big Bang and the black holes did not suffice to predict the rotation of galaxies and the expansion of the universe; to match astronomical data some other theoreticians found it useful to introduce two additional artifacts: dark matter and dark energy.

The problem is that both Newton and Einstein did not really know the nature of space and of gravitation and were completely conscious of the mathemati$\mathrm{cal} /$ geometrical nature of their models and of the caution to be used when trying to explain with mathematics the nature of the universe.

The pessimistic view of the present introduction is that an artifact, ether, has been eliminated, substituting it with an unreal vacuum and inventing a new bushel of artificial objects to fill the unknown.

The optimistic one is that new data are coming to light from nuclear and astronomical investigations, new computational tools and algorithms are available and over all new ideas are circulating worldwide with the speed of light. 


\section{The Source of Light and the Luminiferous Ether}

The Sun emits $4382 \times 10^{9} \mathrm{~kg} / \mathrm{s}$ in the form of light and the particle mass loss is estimated $1374 \times 10^{9} \mathrm{~kg} / \mathrm{s}$, that might appear very large but is only $9.13 \times 10^{-14}$ of the mass of the Sun per year.

This mass/energy is emitted in discrete amount known as quanta from the surface of the Sun.

The intensity and spectral distribution of surface radiation follows the black body model that, after Plank (1904), has been assumed as the emission law of thermal radiation from matter, no apparent relation with the emission from the internal core of matter even if, in both cases, the original mechanism should be the same with light/energy emitted by atoms by "quantum leaps" of electrons between fixed energy levels.

In Fermi model of $\beta$ decay electrons are emitted together with a neutrino by nuclear proton-neutron transformations, followed by rearrangement of nuclear bonds to a more stable configuration with the emission of $\gamma$ rays.

In previous works [11] [12] [13], we have proposed that the behavior of all atoms, stable and unstable isotopes, should have this mechanism, with the electrons being shoot out and recaptured by nuclei following the trajectories of known orbital.

If the shooting energy is too high as in unstable nuclides or if external energy is added, as in the photoelectric effect, the electron is emitted from the atom.

The neutrino emission has been introduced by Fermi to justify the kinetic energy distribution of $\beta$ particles by unstable isotopes, but in our extended view of the atom, the neutrino is emitted by all atoms and can be thought as a cold/ warm particle or simply as a photon having the temperature of the emitting source.

$$
\begin{aligned}
& \beta^{-} \text {emission: } n \stackrel{k_{1}}{\longleftrightarrow} p+\beta^{-}+v \\
& \beta^{+} \text {emission: } p \stackrel{k_{2}}{\longleftrightarrow} n+\beta^{+}+v
\end{aligned}
$$

Orbital electron capture: $p+\beta^{+} \stackrel{k_{3}}{\longleftrightarrow} n+v$

and the electron-positron annihilation reaction with the production of two $\gamma$ photons having energy of $0.511 \mathrm{MeV}$ (megaelectron volt) each, equal to the rest energy of an electron

$$
\beta^{-}+\beta^{+}=2 \gamma
$$

In [1], we introduced the hypothesis that the energy of the nuclear bond is two annihilation reactions $(2044 \mathrm{MeV})$ and computed the number of bonds of light nuclides: surprisingly this number is perfectly related to the combinations without repetitions of $p, n$ nucleons, supporting the representation of nuclei in continuous dynamic $p$ - $n$ transformations $(10,11)$.

In this view, the experimental evidence that the universe we know is made up of nuclides having a particular $n-p$ distribution, leads to an obvious consequence of fundamental interest. 
Based on n-p distribution of existing nuclides, in [1] we have computed from Equation (10) $k_{1}, k_{2}$ and $k_{3}$ and considered them as the primary universal constants because they represent, with a determination near one hundred percent, the $\mathrm{n}$ - $\mathrm{p}$ distribution of nuclides present in our universe:

$$
k_{1}=0.0009625, k_{2}=4.71554 E-06, k_{3}=0.00105382
$$

The computation $\mathrm{d} N / \mathrm{d} t=-\mathrm{d} P / \mathrm{d} t$ yields negative values for $\beta$ - emitters and positive ones for $\beta^{+}$emitters the higher values corresponding to most active emitters.

This model with these constant allow the computation of the original emission of radiation by matter and, surprisingly again, this flux is for all nuclides almost constant per gram of matter and equal to $F_{o}=6.668 \mathrm{E}+20$, that is the number of neutrino/photons emitted per gram per second $\left(v \cdot \mathrm{g}^{-1} \cdot \mathrm{s}^{-1}\right)$.

This value is quite different from surface emission and as reported in [13], this flux is generated inside bodies, makes atoms and molecules vibrate and finally escapes from the surface (even from adiabatic surface) in the form of neutrino or graviton.

The neutrino is of particular interest because it is emitted by matter and can pass the nucleons without interactions: a body subject to a neutrino flux is therefore subject to an unusual momentum balance, between incoming and emitted neutrinos, that causes a pull on the body toward the incoming neutrino flux.

This subtle phenomenon allows the writing of Newton's law from basic principles, relating gravity uniquely to properties of matter [11] [12].

This simple physical representation of gravity allows the computation of the "universal" gravitational constant $G$ from $F_{o}$ and from nuclides properties:

$$
G=F_{o} \mu r_{n}^{2} c / 4 m_{o}
$$

where $\mu$ is the neutrino mass $r_{n}$ and $m_{o}$ are the nucleon radius and mass, $c$ the speed of light.

The Gauss constant $G$, directly related to $F_{o}$, therefore carries within it all incertitude of $F_{o}$, due to its feeble variation with matter composition.

The application in [14] to the Earth and to the Milky Way fits experimental values without the need of artefacts like dark energy and dark matter.

In addition it has been shown [15] that the emission of neutrino from the Earth and from the Sun can explain the change in Earth revolution time.

The presence of this flux of neutrino/graviton has an enormous consequence for the definition of the mechanism of light transmission and for the comprehension of what vacuum or the luminiferous ether is.

In spite the great quantity of light emitted by the Sun and its importance for our life, the Sun loses with the flux of neutrino $(\mu=1.55277 \mathrm{E}-36 \mathrm{~g} / \mathrm{n}) 2.05949$ $\mathrm{E}+18 \mathrm{~g} \cdot \mathrm{s}^{-1}$ that is six order of magnitude surface radiation.

We must not complain for the life of the Sun that is certainly shorter than with surface radiation alone, because this is the fee we have to pay for our every year round trip: no perpetual motion is allowed in nature. 
The concentration of emitted matter near the Sun is high: using Gauss theorem, we can easily compute the flux normal to the surface and then divide by surface and velocity of light to have concentration.

We get a flux $3.3832 \mathrm{E}-05 \mathrm{~g} \cdot \mathrm{cm}^{-2} \cdot \mathrm{s}^{-1}$ and a concentration of the order of $1.1288 \mathrm{E}-15 \mathrm{~g} \cdot \mathrm{cm}^{-3}$ and this corresponds to the gravity acceleration on the surface of the Sun; we know however that for some diameter in the proximity of the surface there is an important isotropic tangential flux that, on the surface, is the same order of magnitude of the normal one.

On the surface of the Earth the flux of matter from the Sun is $7.3238 \mathrm{E}-10$ $\mathrm{g} \cdot \mathrm{cm}^{-2} \cdot \mathrm{s}^{-1}$ and the concentration is $2.4435 \mathrm{E}-20 \mathrm{~g} \cdot \mathrm{cm}^{-3}$ while those originated by the Earth are $1.2272 \mathrm{E}-06 \mathrm{~g} \cdot \mathrm{cm}^{-2} \cdot \mathrm{s}^{-1}$ and $4.0944 \mathrm{E}-17 \mathrm{~g} \cdot \mathrm{cm}^{-3}$ respectively.

With reference to the calculation reported in [14], in Figure 1 and Figure 2 we can draw a graph representing the flux and the concentration of neutrino in and around Earth.

We can see from Figure 1 that the neutrino flux, that is responsible of gravity FiZ, is null in the center of Earth, linearly increases to the surface and then drops with the square of distance.

There is however a component of flux in the Earth and near the surface with zero net value and isotropic in the transverse direction, FiXY whose absolute value is very high inside Earth and is the same order of FiZ on its surface.

The total flux Fit and concentration $\rho$ of neutrino is maximum in the centre and at a distance of four Earth radiuses exceeds two orders of magnitude that due to the Sun.

That is why, on the Earth, the influence of the Sun on the speed of light is almost null and the same can be said for the galaxy: the Earth can be thought as an isolated system dominated for several diameters from its surface by the emission of its mass.

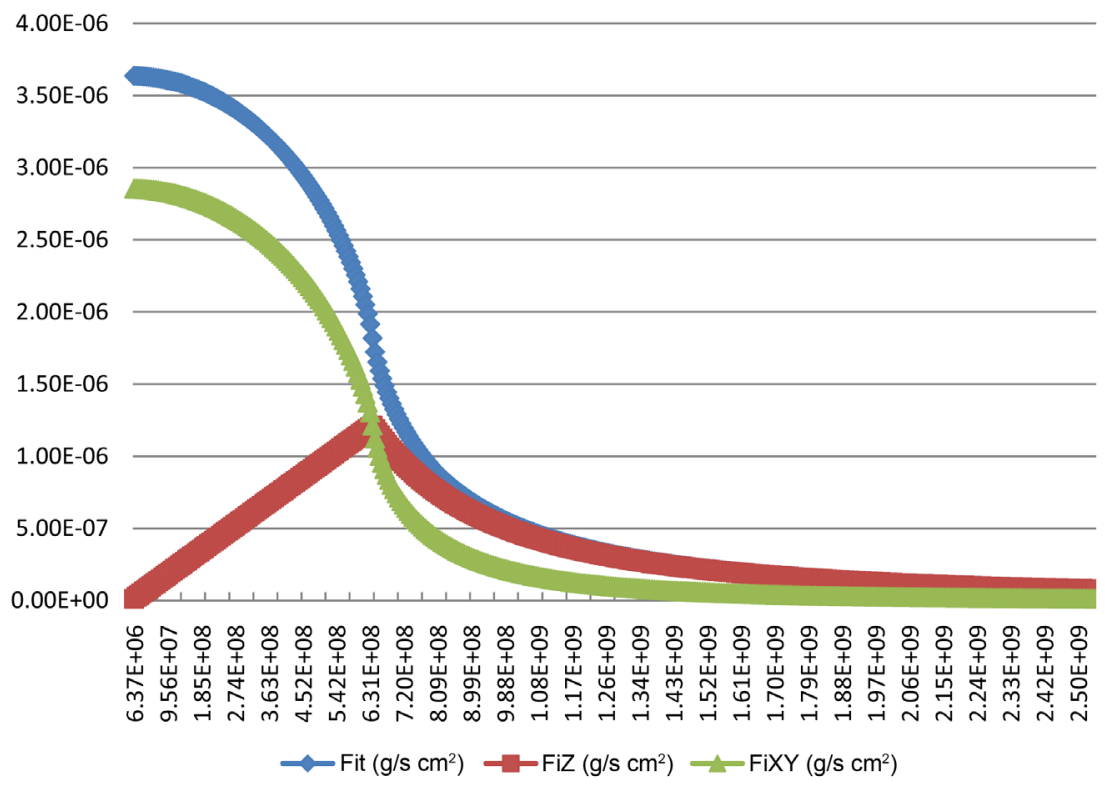

Figure 1. Neutrino FLUX in function of distance from center of Earth $\left(\mathrm{g} / \mathrm{s} \cdot \mathrm{cm}^{2}\right)$. 


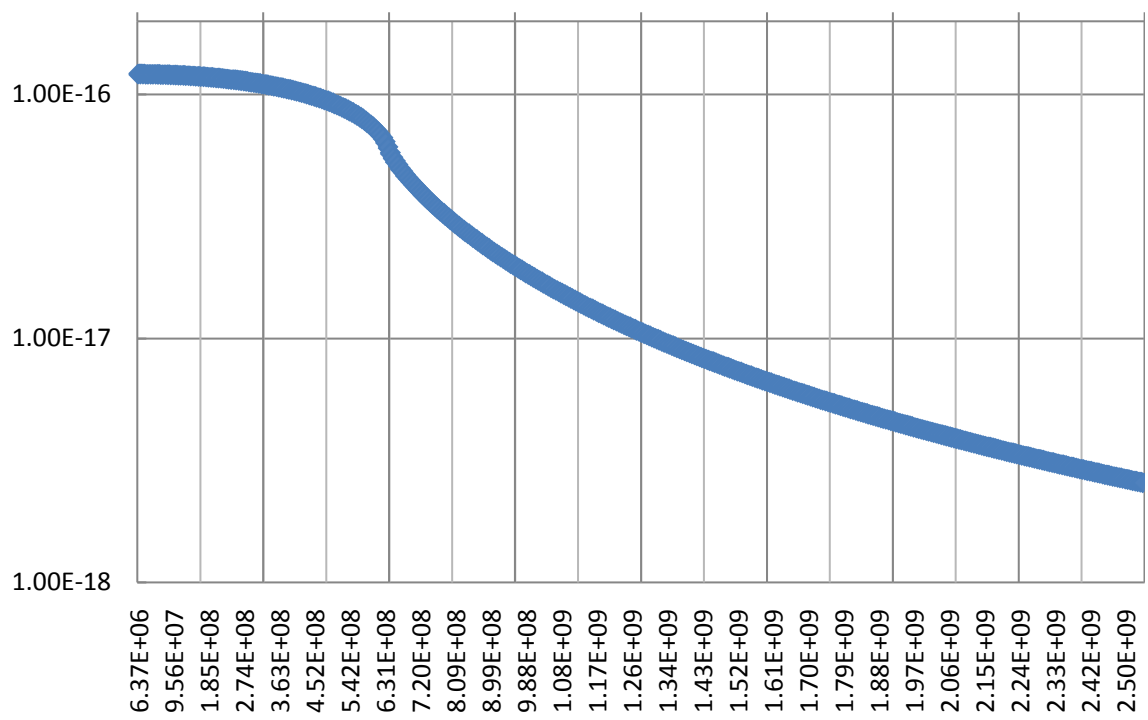

Figure 2. Neutrino concentration $\rho$ in function of distance from center of Earth $\left(\mathrm{g} / \mathrm{cm}^{3}\right)$.

This explains why the Michelson and Morley [1] experiment gave null result and the GPS measurements were able to appreciate the influence of the Earth rotation and not of the Sun [2]-[7], Fresnel-Fizeau effect included.

The predominance of static effect on all gravitational phenomena is also perceived: gravity is not a force transmitted with the speed of light but the result of a balance of neutrino-gravitons entering and exiting the nucleons of a body.

In a point at a fixed distance from the Sun the neutrino flux of the Sun is the same 8 minutes before and after the arrival of a planet, without the need of an infinite speed of transmission: with this respect the effect of time is negligible.

\section{Back to Relativity}

Now that we have perceived the nature of the medium in which light travels, that is not vacuum devoid of matter nor the static ether or its rotating version, we might ask Einstein to help us in the computation of the speed of light in such a medium.

The reason we refer to Einstein is that all papers and newspapers, pro and against him, cite his work as an anticipation of new findings like signals from the space of gravitational waves and black holes, traces of particles waiting to be discovered or new measurements of neutrino and light speed.

To tell the truth, Einstein did not agree with many of the findings that some modern scientists attribute to him.

As for the speed of light, he had some problems from special to general relativity, but finally he honestly recognized the limits of his theory. We cite his own thought taken from his first (1911) paper on general relativity:

"Aus dem soeben bewiesenen Satze, daß die Lichtgeschwindigkeit im Schwerefelde eine Funktion des Ortes ist, läßt sich leicht mittels des Huygensschen Prinzipes schließen, daß quer zum Schwerefeld sich fortpflanzende Lichtstrahlen eine Krümmung erfahren müssen." [9] 
("From the just proved assertion, that the speed of light in a gravity field is a function of position, it is easily deduced from Huygens's principle that light rays propagating at right angles to the gravity field must experience curvature.")

In a subsequent paper in 1912, he concluded that:

"Das Prinzip der Konstanz der Lichtgeschwindigkeit kann nur insofern aufrechterhalten werden, als man sich auf für Raum-Zeitliche-Gebiete mit konstantem Gravitationspotential beschränkt.”[16]

("The principle of the constancy of the speed of light can be kept only when one restricts oneself to space-time regions of constant gravitational potential.")

While this gives an answer to his detractors, we have seen that he provides a way. Using Expressions (7) and (8), to compute the speed and the curvature of light in the presence of a gravitational field.

To maintain special relativity, he imagined regions of space time, as for example a freefalling elevator, in which gravity is null even in a very strong gravitational field.

In addition he imagined that, due to length and time contraction, the speed of light should appear locally the same and hence, following the Equivalence Principle, space time is a patchwork of locally flat frames meshed smoothly to describe a curved space.

From this point of view the centre of the Earth could appear as a perfect zero gravity region for special relativity, even if there we have the maximum concentration of neutrino.

There is no place in the universe devoid of matter, even if we can imagine places where gravity is null or small and, even forgetting the elusive neutrino, the visible part of it remains as cosmic radiation background.

The subtle matter concentration and its variation due to the distribution of gravitation masses was perhaps the medium Einstein thought to apply Freshnel-Huygens's principle.

Now that we can determine the distribution of matter around gravitational masses we can try to compute the change of the speed of light with the distances from the surface of the Earth and estimate the effect of neutrino density.

We can compute following Einstein the relative increase of speed at distance $R$ from the center of mass $M$ with respect of its surface in vacuum:

$$
D_{1}=\frac{C-C_{o}}{C_{o}}=\sqrt{\frac{1+\frac{2 G M}{R C_{o}^{2}}}{1+\frac{2 G M}{R_{o} C_{o}^{2}}}}-1
$$

We can relate this speed variation not to geometry but to the local state of vacuum, e.g. to the neutrino concentration $\rho\left(\mathrm{g} / \mathrm{cm}^{3}\right)$ and, for comparison with Einstein expression, normalize with a coefficient $A$ to the far values where the gravitational component is prevailing:

$$
D_{2}+A=\frac{C-C_{o}}{C_{o}}=\frac{\left(1+\rho_{o}^{0.5}\right)^{0.5}}{\left(1+\rho^{0.5}\right)^{0.5}}-1
$$


For the Earth we can compute $\rho$ as in Figure 2 and obtain $D_{1}$ and $D_{2}$ in Figure 3.

The difference between $D_{1}$ and $D_{2}$ is due to a concentration of neutrino near the surface that is higher than that causing gravity.

The effect is very small: the speed of light at $20.000 \mathrm{~km}$ from the surface of Earth is $15 \mathrm{~cm} / \mathrm{s}$ higher for Einstein and, for the suggested density regression, 90 $\mathrm{cm} / \mathrm{s}$ than on the surface.

We can change the meter and the clock here and there, in order to maintain the speed of light $c$ as a universal constant, but the questions, raised by the new idea of gravity, go beyond relativistic effects with the Gauss constant $G$ fluctuating with $F o$ and the kilogram ageing with time.

Together with relativity, this extended relativity is perhaps the piece of knowledge we need to make some progress in physics.

\section{Conclusions}

The problem of the speed of light has been the obsession of the last century and even today physicians are diving into the problem with the attempt to discover the ultimate laws of the universe.

Einstein paved the way starting from the invariant light speed of special relativity and ending with the geometric-mathematical representation of gravitation of general relativity.

Einstein followers dig into his theory and, having eliminated ether, new ghosts, he never agreed upon, are appearing like phantom energy and matter.

Einstein contrarians try to invalidate the basic principles of relativity, namely the isotropy of light but, in spite of many discussions, a lot of writing and great proposals, the mystery of gravity stands up.

The proposal we have made starts from simple experimental facts on the constitution of matter, with light and neutrino/gravitons emission following similar mechanisms.

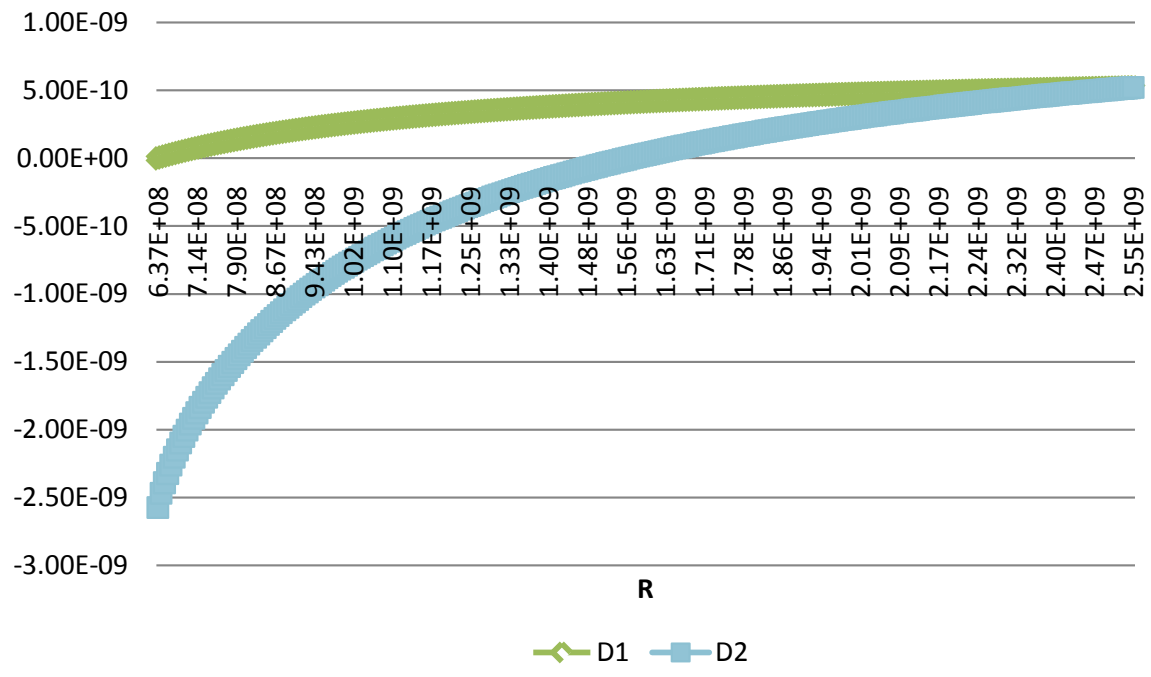

Figure 3. Relative speed of light in function of distance $R$ from earth $D=\left(C-C_{o}\right) / C_{o}$. 
In this changed scenario, a precise physical representation of gravitational effects and light transmission are justified by a better understanding of light experiments, together with the simplification and a more realistic view of our universe.

\section{References}

[1] Michelson, A.A. and Morley, E.W. (1887) The Relative Motion of the Earth and the Luminiferous Ether. The American Journal of Science, 34, 333-345.

https://doi.org/10.2475/ajs.s3-34.203.333

[2] Demjanov, V.V. (2010) Physical Interpretation of the Fringe Shift Measured on Michelson Interferometer in Optical Media. Physics Letters A, 374, 1110 -1112.

[3] Demjanov, V.V. (2012) The Compatibility of Non-Negative Outcome of Michelson \& Morley Experiment with Lorentz-Invariant Transformation of the Light Speed in Moving Optical Media. Admiral Ushakov University, Novorossisk.

[4] Shaff, J. (2012) The Nature of Space and of Gravitation. Journal of Modern Physics, 3, 714-749. https://doi.org/10.4236/jmp.2012.38097

[5] Gift, S.J.G. (2012) GPS and the One-Way Speed of Light. https://cdn.intechopen.com/pdfs-wm/39778.pdf

[6] Schreiber, B. (2007) The Final Analysis of the Michelson-Morley Aether Drift Experiment and the End of the Aether Concept. The General Science Journal. http://www.gsjournal.net/old/science/schreiber34.pdf

[7] Croca, J.R. (2000) Experimental Proposal for Determination of One-Way Velocity of Light with One Single Clock. http://cfcul.fc.ul.pt/biblioteca/online/pdf/josecroca/poincare-curse.pdf

[8] Einstein, A. (1916) Die Grundlage der allgemeinen Relativitastheorie. [The Foundation of Generalized Theory of Relativity]. Annalen der Physik, 354, 769-822. https://doi.org/10.1002/andp.19163540702

[9] Einstein A. (1911) Uber den Einfluss der Schwerkraft auf die Ausbreitung des Lichtes. [On the Influence of Gravitation on the Propagation of Light]. Annalen der Physik, 340, 898-908.https://doi.org/10.1002/andp.19113401005

[10] Pound, R.V. and Rebka Jr, G.A. (1959) Gravitational Red-Shift in Nuclear Resonance. Physical Review Letters, 3, 439-441. https://doi.org/10.1103/PhysRevLett.3.439

[11] Donati, G. (2004) Il legame nucleare e il peso del neutrino. [The Nuclear Bond and the Neutrino Weight]. La Chimica e L'Industria, 62-66.

http://www.academia.edu/8243167/

[12] Donati, G. (2006) L'universo invisibile. [The Invisible Universe]. ICPN, 92-97. http://www.academia.edu/8243430/

[13] Donati, G. (2012) The Kinetic Theory of Matter and Perpetual Motion. http://www.academia.edu/8068603

[14] Donati, G. (2015) What Gravity Is. http://www.academia.edu/11425803/

[15] Donati, G. (2017) Rethinking the Earth in the Solar System. Journal of Applied Mathematics and Physics, 5, 631-638. https://doi.org/10.4236/jamp.2017.53054

[16] Einstein, A. (1912) Lichtgeschwindigkeit und Statik des Gravitationsfeldes. [The Speed of Light and the Static Gravitational Field]. Annalen der Physik, 343, 355-369.https://doi.org/10.1002/andp.19123430704 